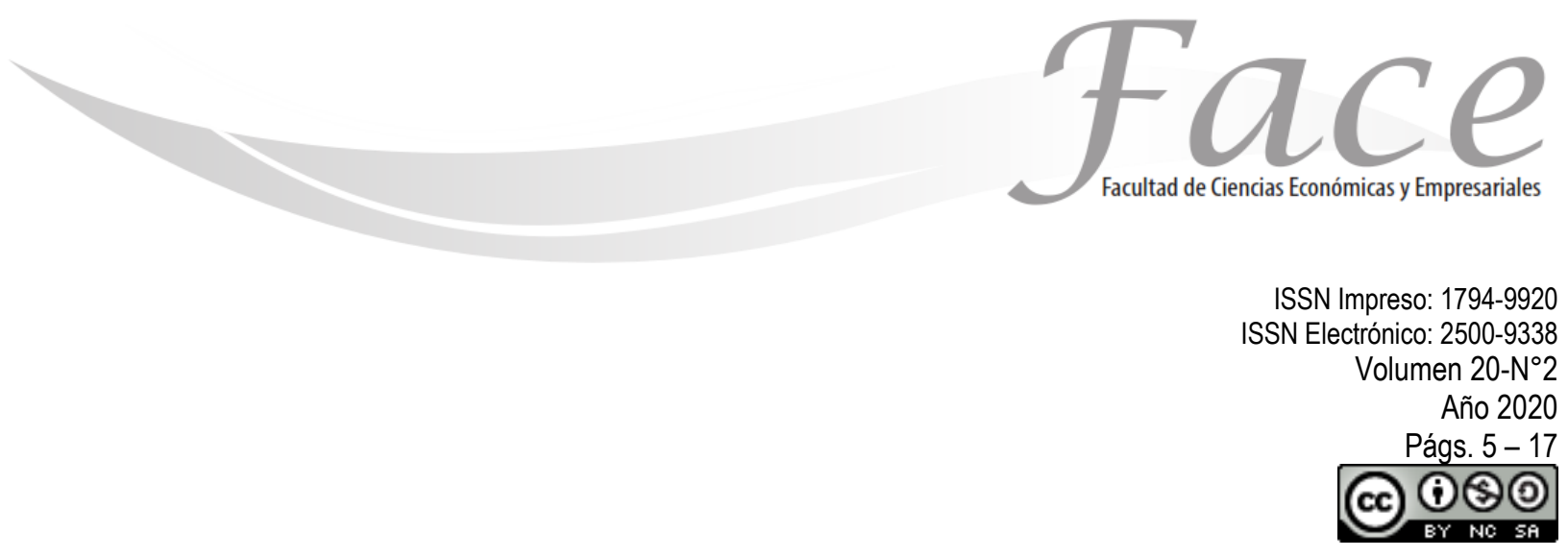

\title{
CRM UNA ESTRATEGIA DE FIDELIZACIÓN DE CLIENTES EN LAS IES. ÁREA DE EDUCACIÓN CONTINUA
}

*

\author{
Cerón Islas Arlen* \\ Enlace ORCID: https://orcid.org/0000-0003-3982-5053 \\ Cerón Islas Heidy ** \\ Enlace ORCID: https://orcid.org/0000-0003-3373-6949 \\ Cortés Servín Edgar Antonio ** \\ Enlace ORCID: https://orcid.org/0000-0002-2039-5885
}

Fecha de Recepción: Agosto 25 de 2020

Fecha de Aprobación: Noviembre 29 de 2020

\section{Resumen:}

El presente tiene por objetivo identificar los beneficios y contribuciones de la implementación de la estrategia de Marketing de Relación, Costumer Relationship Management (CRM) y sus complementos, en una institución de educación con la finalidad de modelar el tipo de cliente para la atención personalizada.

Aplicando una investigación de carácter cualitativo descriptiva, mediante entrevista abierta y un análisis FODA para identificar la problemática; se realizó una propuesta de solución con base en la aplicación de las teorías del Marketing de Relación, CRM y sus complementos; buscando la consolidación y fidelización del mercado con miras a consolidación en el mercado.

Palabras clave: Mercadotecnia, CRM, Fidelización

\footnotetext{
* Doctora en Planeación Estratégica y dirección de tecnología. Profesora Investigadora del Instituto de Ciencias Económico Administrativas, México. Universidad Autónoma del Estado de Hidalgo, México. Contacto: arlenc@uaeh.edu.mx

** Doctora en Planeación Estratégica y dirección de tecnología. Profesora por asignatura del Instituto de Ciencias Económico Administrativas, México. Universidad Autónoma del Estado de Hidalgo, México. Contacto: heidy_ceron6257@uaeh.edu.mx

*** Pasante de la Licenciatura en Mercadotecnia del Instituto de Cienbocias Económico Administrativas, México. Universidad Autónoma del Estado de Hidalgo, México. Contacto: tonycorssen@gmail.com
} 


\section{CRM A CUSTOMER LOYALTY STRATEGY IN IES. CONTINUING EDUCATION AREA}

\section{Abstract:}

The objective of this document is to identify the benefits and contributions of the implementation of the Relationship Marketing strategy, Costumer Relationship Management (CRM) and its complements, in an educational institution in order to model the type of client for personalized attention.

Applying a descriptive qualitative research, through an open interview and a SWOT analysis to identify the problem; a solution proposal was made based on the application of Relationship Marketing theories, CRM and its complements; seeking the consolidation and loyalty of the market with a view to consolidation in the market.

Keywords: Marketing, CRM, Loyalty.

\section{CRM UMA ESTRATÉGIA DE FIDELIZAÇÃO CLIENTES NO IES. ÁREA DE EDUCAÇÃO CONTINUADA}

\section{Resumo:}

O objetivo deste documento é identificar os benefícios e contribuições da implementação da estratégia de Marketing de Relacionamento, Gestão de Relacionamento com o Cliente (CRM) e seus complementos, em uma instituição de ensino, a fim de modelar o tipo de cliente para atendimento personalizado.

Aplicação de uma pesquisa qualitativa descritiva, por meio de entrevista aberta e análise SWOT para identificação do problema; foi feita uma proposta de solução baseada na aplicação das teorias de Marketing de Relacionamento, CRM e seus complementos; buscando a consolidação e fidelização do mercado com vistas à consolidação no mercado.

Palavras-chave: Marketing, CRM, Lealdade. 


\section{INTRODUCCIÓN:}

La principal problemática a la que se enfrentan hoy en día empresas e instituciones que están en el mercado ya desde hace mucho tiempo es que, si no fueron creciendo a la par de los líderes de mercado en su rubro, el adaptar sus procesos a nuevas tecnologías que les permitan ser más eficientes, precisos y seguros, a la hora de realizarlos o de tomar decisiones asegura su declive en la industria y en algún punto desaparecer. Esto es en todas las industrias, pero en la educación es primordial para las instituciones estar actualizados en todo sentido, ideológica, pedagógica y tecnológicamente, además de conocer a la perfección al mercado al que se dirigen para así desarrollar productos y un servicio específico que le permita ser atractivo para su mercado cautivo y potencial.

El área de educación continua de esta Universidad es el organismo que ofrece, además de a la comunidad Lasallista, al público en general los servicios de certificación y actualización de conocimientos en diversas materias. Actualmente atienden, capacitan y certifican a más de 600 personas externas en promedio, con una oferta educativa de más de 50 productos entre cursos, diplomados y talleres.

La presente investigación pretende aportar la información y fundamentos necesarios al sector educativo para entender la importancia de la adopción de la estrategia de marketing de relación CRM, aceptándolo como una herramienta de desarrollo estratégico que potencializa en diversos sectores a las instituciones y áreas dependientes que 10 apropian. Por lo que el objetivo principal es identificar los beneficios y contribuciones de la implementación de la estrategia de marketing de relacional, Costumer Relationship Management (CRM) que optimizará la relación entre las instituciones de educación por medio del área de Educación Continua y sus clientes.
En ese orden de ideas, el área de educación continua de la Universidad La Salle de Pachuca, no cuenta con herramientas tecnológicas que le permitan tener un flujo constante, veras y actualizado de información de sus clientes. Impidiéndole ofrecer una atención más personalizada y de impacto en los alumnos y/o públicos que adoptan sus servicios. Así como diseñar estrategias de venta y promoción que impacten a los segmentos a los que se dirige.

Las adopciones de este tipo de filosofías enfocadas al cliente ayudan al área a poder atender y entender de manera adecuada a su mercado cautivo, permitiendo no sólo la fidelización del mismo, sino, la adopción pronta y sistematizada de nuevos mercados.

\section{MARCO TEÓRICO:}

\section{Marketing Relacional}

De acuerdo con Gröroos (1999) el marketing relacional es un proceso en cual identifica, establece y mantiene las relaciones con consumidores de interés, contemplando los objetivos de las áreas involucradas mediante un intercambio y cumplimiento de las metas, por lo que este autor menciona que el producto, precio, lugar y promoción precede del marketing de relaciones, sin embargo para este proyecto de marketing relacional se define como una estrategia lógica en la que se realiza un orden de la comercialización de bienes y servicios, siempre tomando en cuenta la necesidad de los clientes rentables de manera colectiva y así lograr los objetivos de las partes involucradas.

\section{Marketing Transaccional}

El paradigma tradicional del marketing se caracteriza por una orientación de tipo transaccional, la tesis central es que se circunscribe a la mezcla de marketing, a través de la mixtura de cuatro variables convence al consumidor para que compre un 
determinado producto (Gummesson, 2002; Citado por Córdoba, 2009, p. 8).

Entre las debilidades del paradigma transacción está la fuerte orientación y concentración hacia la mezcla de marketing, la cual indica que, mediante la apropiada mixtura de cuatro variables, se puede conseguir unir sobre el consumidor para que compre un determinado producto. En tal sentido, la mezcla de marketing presenta, en realidad, una orientación más centrada en el producto, que en los consumidores (Laycock, 1991; Gröroos, 1989; Citado por Córdoba, 2009, p. 9).

En la imagen 1 se muestra las características del marketing transacciones vs marketing relacional según los autores Martínez y Luna (2007). sistemas de información integrados, teniendo como elemento básico operativo la información continua de los alumnos (Román, et al 2005, p. 40).

Díaz (2015) describe las estrategias de CRM como una estrategia de entendimiento, anticipación y respuesta rápida y efectiva para las necesidades de los clientes actuales y potenciales, ocasionando con esto que el nivel de relación entre ambas partes se dirija al incremento. El error más común que cometen las empresas que lo implementen es creer que la estrategia de CRM es tan solo un software que les permita obtener información de los clientes y que por sí solo va a cumplir con las expectativas de los usuarios, así como lograr las metas y resultados esperados por la organización. Las buenas implementaciones de la estrategia cambian la gestión de información combinada con datos operacionales y herramientas analíticas que otorguen el conocimiento necesario para las organizaciones auxiliares en la toma de decisiones.

\section{Imagen 1}

Características de marketing transacional y relacional.

\begin{tabular}{l|l}
\hline \multicolumn{1}{|c|}{ Marketing Transaccional } & \multicolumn{1}{c}{ Marketing Relacional } \\
\hline Centrado en una sola venta. & Centrado en la retención del cliente. \\
\hline Centrado en las características del producto. & Centrado en los beneficios del producto. \\
\hline $\begin{array}{l}\text { Orientado al corto plazo. } \\
\text { Poco énfasis en el servicio al cliente. }\end{array}$ & Orientado a largo plazo. \\
\hline $\begin{array}{l}\text { Compromiso limitado con la de alcanzar las } \\
\text { expectativas del cliente. }\end{array}$ & $\begin{array}{l}\text { Alto compromiso con la idea de alcanzar las expectativas del } \\
\text { cliente. }\end{array}$ \\
\hline $\begin{array}{l}\text { Contacto discontinuo con el cliente. } \\
\text { La calidad es una cuestión propia del departamento al cliente. } \\
\text { de producción. }\end{array}$ & Contacto continuo con el cliente. \\
\hline
\end{tabular}

Fuente: Elaboración propia, 2018, a partir de Martínez y Luna, (2007; p 43)

\section{CRM (Costumer Relationship Management)}

El CRM (Costumer Relationship Management) puede tener una enorme potencia e influencia en los procesos de la enseñanza y aprendizaje, si se define como una estrategia basada en la búsqueda de la optimización

de las relaciones con los alumnos para mejorar su rendimiento y fidelidad, además en la utilización de la información derivada de estas relaciones para dirigirlo. Adoptando este concepto, podemos pensar en tener toda la organización interconectada a través de
Espinosa (2012) y Harding (2004) establecen que el término se encuentra estrechamente relacionado con la gestión de las relaciones que genera la empresa con el cliente existente, lo que implica que exista una relación personal entre cliente y profesional. Se plantea que cuando una empresa tiene la capacidad de poder ofrecer una cartera de servicios amplia al cliente es un camino para ascender, puesto que quienes trabajan en posiciones más altas suelen tener una gran capacidad para la venta cruzada. 
En particular, Harding (2004) considera tres tipos de venta cruzada, vender múltiples servicios a un único cliente, vender a distintas unidades empresariales de la misma cuenta y vender servicios a la misma cuenta en distintas regiones, para las empresas una de las principales razones para ofrecer una amplia gama de servicios es cubrir estas capacidades, al generar un mayor poder de compra y tener una imagen.

\section{Satisfacción del cliente}

Kotler (2002) expone que las sensaciones de placer 0 satisfacción que provienen de un consumidor, conllevan una perspectiva diferente, ya que este sentimiento se origina en una persona de forma obligada al comparar el desempeño o resultado de lo percibido de un producto o servicio con sus expectativas al ser o no cumplidas.

\section{CRM y su importancia en la toma de decisiones.}

De acuerdo con Valle, et al (2017, p. 111) el CRM busca la eficiencia de los procesos en la otra de decisiones, buscando dos factores; uno, buscar la fidelización del cliente y dos, encontrar la rentabilidad de la empresa en el esfuerzo de complacer a su mercado.

Definiéndolo de manera generalizada se puede decir que el CRM busca la total satisfacción del cliente a la par de la rentabilidad de la empresa, a partir de la construcción de una base de datos que contenga datos fidedignos que permita modelar el cliente y sus necesidades.
Valdés (2007) define a la base de datos como el conjunto de datos organizados y relacionados entre sí que son recolectados y aprovechados por los sistemas de información de la empresa o negocios para su beneficio.

Entre las principales características de los sistemas de base de datos menciona las siguientes:

1. Independencia lógica y física de los datos.

2. Redundancia mínima.

3. Acceso concurrente por parte de múltiples usuarios.

4. Integridad de los datos.

5. Consultas complejas optimizadas.

6. Seguridad de acceso y auditoría.

7. Respaldo y recuperación.

8. Acceso a través de lenguajes de programación estándar.

Áreas y procesos en los que el CRM aporta, son los que se visualizan en la Imagen 2; los cuales son principalmente gestión de cuentas y contactos, servicio al cliente y E-Commerce.

Los tres componentes del CRM (Colaborativo, Operacional y Analítico) tienen la capacidad de operar independientemente sin embargo juntos tienen la oportunidad de soportar la infraestructura de CRM por medio de sus elementos, tales como la interacción, integración, información e inteligencia. Los tres componentes se apoyan para poder desarrollar estrategias eficientes de negocio (Grupo 5, 2006). 


\section{Imagen 2}

Áreas y procesos en los que el CRM aporta

\begin{tabular}{|c|c|c|}
\hline $\begin{array}{l}\text { Gestión de cuentas \& } \\
\text { contactos }\end{array}$ & Servicio al cliente & E-Commerce \\
\hline Perfil del contacto & Análisis de incidencias & Shopping Cart \\
\hline Histórico de actividades & Asignación de incidencias & Pedidos on line \\
\hline Cuadro organizativo & Registro de incidencias & Gestión de oportunidades \\
\hline Relaciones de negocio & $\begin{array}{l}\text { Registro de peticiones de } \\
\text { servicio }\end{array}$ & $\begin{array}{l}\text { Generador de oportunidades } \\
\text { de internet }\end{array}$ \\
\hline Gestión de pedidos & Herramientas self-service & Conexión con partners \\
\hline Generador de propuestas & Marketing & Business Inteligence \\
\hline $\begin{array}{l}\text { Gestión de cuentas de } \\
\text { internet }\end{array}$ & Planificador de campañas & Reporting predefinido \\
\hline Integración Outlook & Gestión de eventos & Gestión de listados \\
\hline Calendario nativo & Enciclopedia de marketing & Gestión de alertas \& alarmas \\
\hline $\begin{array}{l}\text { Sincronización } \\
\text { multiplataforma }\end{array}$ & Literatura & $\begin{array}{l}\text { Monitorización de } \\
\text { productividad real-time }\end{array}$ \\
\hline Gestión de ventas & Base de datos de marketing & \\
\hline Gestión de oportunidades & Análisis ROI de campaña & Tecnologia \\
\hline Forecasting & Integración de MS-Word & $\begin{array}{l}\text { Herramienta rápida de } \\
\text { desarrollo }\end{array}$ \\
\hline \multicolumn{2}{|l|}{ Análisis de ciclo de venta } & Soporte SQL multiplataforma \\
\hline \multicolumn{2}{|l|}{ Estadisticas de venta } & $\begin{array}{l}\text { Sincronización de datos a } \\
\text { través de diferentes } \\
\text { plataformas }\end{array}$ \\
\hline \multicolumn{2}{|l|}{ Reporting de actividad } & $\begin{array}{l}\text { Seguridad multinivel para } \\
\text { usuarios y datos }\end{array}$ \\
\hline \multicolumn{2}{|l|}{ Asignación de territorio } & Sistemas de ayuda on line \\
\hline \multicolumn{2}{|l|}{ Reporting de gastos } & Escalabilidad \\
\hline \multicolumn{2}{|l|}{ Gestión de ventas } & $\begin{array}{l}\text { Integración on line con otras } \\
\text { aplicaciones }\end{array}$ \\
\hline \multicolumn{3}{|l|}{ Telemarketing / Televenta } \\
\hline \multicolumn{3}{|l|}{ Planificar de llamadas } \\
\hline \multicolumn{3}{|l|}{$\begin{array}{l}\text { Guiones de apoyo } \\
\text { (smartscript) }\end{array}$} \\
\hline \multicolumn{3}{|l|}{ Histórico de llamadas } \\
\hline \multicolumn{3}{|l|}{ Auto-dialing } \\
\hline \multicolumn{3}{|l|}{ Análisis de productividad } \\
\hline Literatura & & \\
\hline
\end{tabular}

Fuente: García, 2001; p. 108

Blasco y Otero (2008), señalan que la investigación cualitativa estudia la realidad en su contexto natural y cómo sucede, sacando e interpretando fenómenos de acuerdo con las personas implicadas, por lo cual en el presente estudio se realizaron entrevistas abiertas las cuales se fundamentaron en un listado general de contenido, de esta manera el entrevistador tiene la total flexibilidad para desarrollar y/o ampliar la entrevista a conveniencia y beneficio; produciendo datos descriptivos: las palabras habladas 0 escritas y la conducta observable.
Como lo menciona Hernández, et al (2010) "Por otro lado, en la indagación cualitativa, los instrumentos no son estandarizados, en ella se trabaja con múltiples fuentes de datos, que pueden ser entrevistas, observaciones directas, documentos, material audiovisual, etc." (p. 409). 
El tipo de investigación a realizar es de carácter cualitativa descriptiva, con base en lo anterior, se realizó una entrevista abierta con los integrantes del área de Educación Continua de la Universidad La Salle de Pachuca para conocer, entre otros temas, los procesos y formas en las que:

\section{1.- Identifican un cliente.}

\section{2.- Los canales de comunicación por los que} interactúan.

\section{3.- Cómo se registra un cliente.}

4.- Cuál es el protocolo de atención.

5.- Lanzamiento de cursos, diplomados y talleres.
El método de recolección es de tipo no experimental, es un estudio descriptivo, ya que la idea es plantear un panorama amplio y detallado de lo ocurrido o establecido en la situación específica a estudiar, se aplicaron 2 instrumentos para recoger información como las entrevistas a los responsables del área de vinculación; así mismo se realizaron observaciones in situ que permitieron describir la problemática principal para el desarrollo de la investigación que como ya se menciona es la falta de herramientas de comunicación permanente con el cliente dado el no contar con información precisa de la tipología de cliente.

De acuerdo con la información obtenida por la herramienta de investigación, es que se identifican los siguientes resultados:

\section{Imagen 3}

\section{Resultados de entrevista abierta}

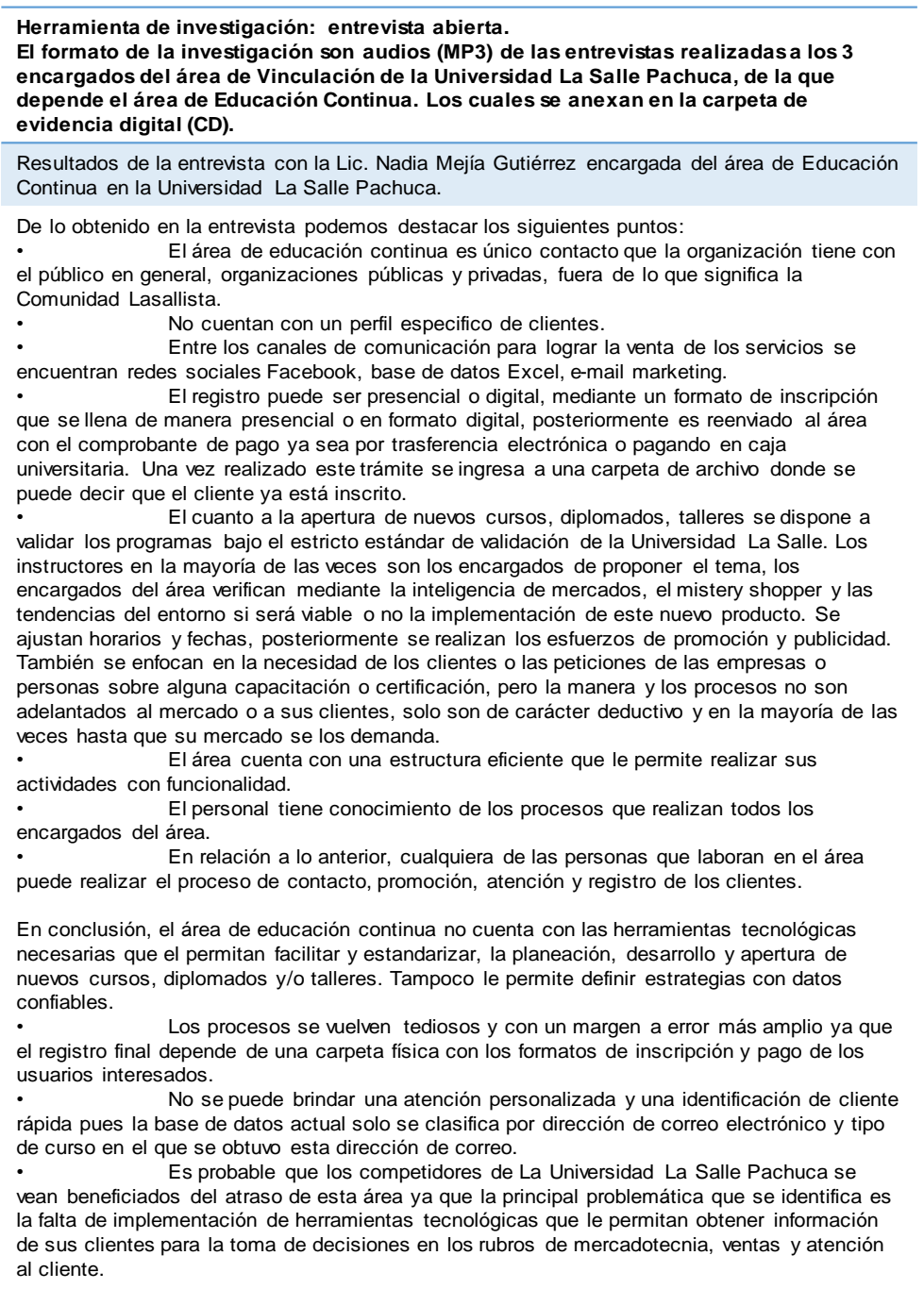


En la observación in situ se realizó un análisis FODA con los siguientes resultados:

\section{Imagen 4}

FODA del área de Educación Continua de la Universidad La Salle de Pachuca

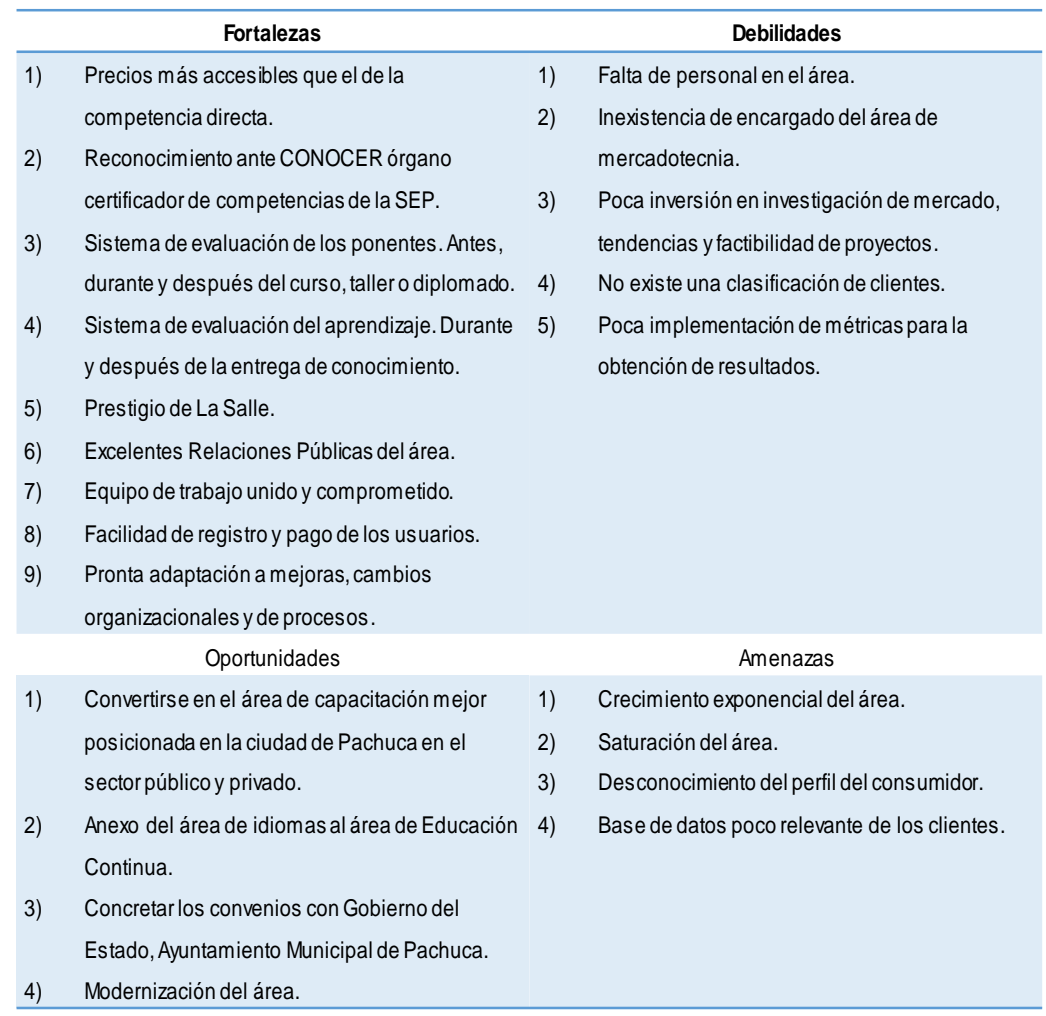

Fuente: elaboración propia, 2020

\section{RESULTADOS Y DISCUSIÓN:}

El área de educación continua no cuenta con las herramientas tecnológicas necesarias que el permitan facilitar y estandarizar, la planeación, desarrollo y apertura de nuevos cursos, diplomados y/o talleres. Tampoco le permite definir estrategias con datos confiables.
- Los procesos se vuelven tediosos y con un margen a error más amplio ya que el registro final depende de una carpeta física con los formatos de inscripción y pago de los usuarios interesados.

- No se puede brindar una atención personalizada y una identificación de cliente rápida pues la base de datos actual solo se clasifica por dirección de correo electrónico y tipo de curso en el que se obtuvo esta dirección de correo. 


\section{8.- Clasificación e identificación de clientes.}

9.- Apoyo como recordatorio para estrategias de fidelización en fechas importantes (aniversarios de inscripción, cumpleaños, etc.).

10.- Información adecuada para ventas y post-ventas personalizadas.

11.- Permite una gestión controlada.

12.- Aumenta la productividad.

\section{Propuesta mercadológica}

Dado lo anterior se puede deducir que es necesario la implementación interna de un sistema tecnológico que permita implementar una estrategia de Social CRM está totalmente ligada al profundo conocimiento de los datos generales de los usuarios ayudado del uso de herramientas tecnológica: el uso de las estrategias que las empresas pueden aplicar para generar un mayor valor en su oferta y una mejor interacción con sus clientes, interactuando más allá de sólo intercambio de likes y tuits, pasando las marcas de hacer transacciones a relaciones.

Implementación de la propuesta de marketing relacional, CRM.

El proceso de implementación del CRM se desarrollará a partir de los procesos de contacto, registro, curso y finalización del o los programas que llegue a utilizar el cliente. Esto incluye la etapa de servicio, ventas y postventa.

La implementación del CRM estará regida por tres ejes de cambio:

Personal: Indispensables para la aplicación de la estrategia, ya que conforman no solo nuestro contacto con el cliente, sino, que depende de este que el CRM funcione y se utilice adecuadamente. Para ello el personal será capacitado de manera que pueda desempeñar sus actividades complementándolas con el uso del CRM a lo largo de las etapas antes mencionadas.
Tecnología: Apoyados de una plataforma conformada por datamar y datamining es como explotaremos al máximo el potencial del CRM; Este contendrá los procesos antes mencionados, además de que proporcionará información relevante que apoyará a la toma de decisiones, por ejemplo: definición del contenido para cursos futuros, temporadas de mayor venta, efectividad de campañas, venta directa por teléfono, email marketing, marketing directo, ticket promedio por cliente, frecuencia de compra.

Como complemento tecnológico, facilitador de procesos y estrategia de fidelización, es que se complementara el software con una tarjeta de identificación personalizada, la cual contendrá datos del cliente que ayudaran a su identificación y rápida personalización de su atención, sea esta presencial o no; fácil acceso a instalaciones y eventos, así como apoyar a la identificación y sentido de pertenencia del cliente con nuestra marca.

Componentes del eje de tecnología:

1. Escáner lector de barras, QR; en pantalla y en impresos. Inalámbrico, de infrarrojo.

El lector tendrá la capacidad de registrar el código asignado al cliente en su base de datos esto lanzara en pantalla el perfil del cliente con las diferentes ventanas de información relevante que se ha generado.

2. Tarjetas de fidelización:

Contendrán la información que requerimos del cliente para los procesos, código de barras o QR, así como número de cliente. También contendrá información para la identificación en los diferentes campus y eventos que la Universidad convoque: Nombre, edad, ocupación y fotografía con vigencia. Además de incluir fecha de renovación con amplitud de 3 años. No servirá como identificación oficial ante ningún órgano 0 institución gubernamental, ni para ningún proceso que no competa a la Universidad Privada.

3. CRM software:

El software deberá de ser diseñado bajo la idea de ser funcional, práctico y claro, pero sin perder el estilo vanguardista e innovador de La Universidad. Se planea producir una plataforma que permita el acceso por cuenta de usuario, esto con respecto a 
los encargados del área. (Máximo 5 cuentas). De esta manera se verá en las gráficas el tiempo de actividades por usuario, determinando quién hizo qué actividad en que tiempo. Permitiendo la evaluación y desempeño de los empleados en la plataforma.

\section{Para el prototipo base se pretende que se conforme de cuatro áreas clave:}

Área 1 - Clientes: contendrá todos los números de usuarios ligados a sus perfiles, contendrá una barra de búsqueda y un botón de agregar en el que se podrá integrar a un nuevo cliente.

El perfil de cada uno de los clientes nos arrojara su perfil con todos los datos personales en la primera pestaña, además de un botón de agregar a evento. Esto también deberá generarse en una determinada selección de usuarios. Que nos enviara a la segunda área del menú principal.

La segunda pestaña nos mostrara los cursos, diplomados, talleres, en los que ha participado, así como tendrá una liga directa a correo electrónico para enviar publicidad 0 promociones personalizadas. Esta acción nos enviara al área 3. La tercera pestaña nos arrojara gráficas de información como, el tiempo de consumo, antigüedad, inversión anual en el área, entre otros.

Área 2 - Cursos, Diplomados, Talleres: en este espacio se realizarán las inscripciones a los nuevos cursos, diplomados y talleres. Tendrá la capacidad de agregar y desagregar eventos, con caratula del curso que funcionará como botón de entrada al evento.

Dentro de cada de los eventos vendrá su breve descripción, la escaleta normal de cada uno, se registrarán todos los integrantes por orden alfabético, podrá agregar el formato de inscripción y el comprobante de pago. Sera completamente editable a manera de definir sus fechas, contenido, docente que imparte, tema y clasificación. También podrán visualizarse por mes de inicio, marcando de esta manera las carteleras y organizando así el trabajo.
También se podrá agregar la calificación obtenida al área por curso, al docente y el nivel de satisfacción, que se practica en cada termino de diplomado, curso 0 taller.

Área 3 - Promoción: en este espacio se podrá visualizar un botón de nueva campaña donde te dará la opción de seleccionar de entre la base de datos ya categorizada los usuarios por características que más te convenga para el producto elegido, o de entre los cursos y talleres que están en curso, así te arrojara solo a los clientes que cumplen con ese perfil, podrás enviarles un correo electrónico personalizado con su nombre a cada uno, y o podrás generar una listado de clientes a los cuales podrás llamar para hacer la venta directa, tendrá la opción de marcarlos como realizado, no realizado, dar seguimiento. Así sabrás y evitaras enviar repetidas veces la información al mismo contacto o llamar impertinentemente cunado ya recibiste una respuesta.

Área 4 - Gráficos: en esta área se encontrarán graficas de análisis de toda la información, visualizables por eventos, por clientes, por periodo de tiempo específico. Encontrarás métricas y resultados que apoyaran en la toma decisiones, como, cantidad de usuarios, rendimiento de campañas, actividad semanal por usuario, clientes en tiempo de renovación, deserciones, metas económicas, por curso, por periodo; ingresos por curso, entre otros.

\section{Adecuación de procesos.}

Procesos: Los procesos realizados por el Área de Educación Continua de la Universidad Privada de Pachuca como: registro de nuevos clientes, inscripción a cursos, petición de información, seguimiento de venta, cierre y culminación de curso, dejará de realizarse sin un proceso definido e informal. Ya que en cada uno de estos se genera la interacción entre el cliente y el personal, proporcionándonos la información necesaria para el buen funcionamiento del CRM.

\section{Nota:}

Los procesos de registro y el uso de los datos personal de los clientes dentro del CRM seguirán bajo las 
condiciones y limitantes del aviso de privacidad del cual depende la Universidad.

Registro: El proceso de registro será de manera presencial. De preferencia el día de inscripción al curso o taller, en su defecto en las primeras dos fechas de inicio del curso, diplomado o taller.

Los procesos con los que el CRM y la tarjeta de identificación funcionarán son:

Registro: El registro se realiza con la introducción de los datos en el sistema entrando al Área 1 del CRM el registro de clientes es de manera guiada y clara metiendo los datos personales de la persona al sistema. Agregando una fotografía que se toma en el momento de inscripción, si el cliente desea cambiar la foto únicamente tendrá la oportunidad de cambiarlo para la fecha de inicio de su curso, en su defecto se cambiará hasta la fecha de renovación. La entrega de la tarjeta de identificación será el día de la graduación de su curso, solo en el caso de los diplomados y cursos que duren más de un mes se entregará 30 días después de la fecha de inscripción.

Inscripción a curso: Para la inscripción existen dos opciones:

Primera Inscripción: Se realiza todo el proceso de registro, después se dirige al botón de agregar a evento donde se adjuntará al evento que así sea seleccionado y posteriormente se podrán anexar los formatos de inscripción y el de pago, asegurando así que el registro en el evento esta completado.

Reinscripción: Dependiendo del canal por el que se realice la inscripción (presencial / no presencial) se pedirá la credencial para escanear el código de barras y entrar de inmediato al perfil del cliente, se procede al registro, dando clic al botón de agregar a evento donde se adjuntará al evento que así sea seleccionado y posteriormente se podrán anexar los formatos de inscripción y el de pago, asegurando así que el registro en el evento esta completado.

Respectivamente para el registro no presencial se pide de manera telefónica el número de cliente, que se encuentra en la tarjeta de identificación, se ingresa en el Área 1 de la plataforma, así arroja el perfil completo del cliente, se procede al registro, dando clic al botón de agregar a evento donde se adjuntará al evento que así sea seleccionado y posteriormente se podrán anexar los formatos de inscripción y el de pago, asegurando así que el registro en el evento esta completado.

En el caso que sea por correo electrónico se pide que se envié la foto del código o el número de cliente, cualquiera de los dos es aceptable. Anexado además los formatos de inscripción y de pago. Se procede al registro, dando clic al botón de agregar a evento donde se adjuntará al evento que así sea seleccionado y posteriormente se podrán anexar los formatos de inscripción y el de pago, asegurando así que el registro en el evento esta completado.

Contacto: Se entra al área de promoción (Área 3) donde se selecciona el evento al que se le invitara a la persona a participar de acuerdo con lo señalado por el perfil donde lo clasifique, se procederá a realizar la estrategia de promoción que más convenga al momento 0 en su defecto ambas, dando posteriormente, un tipo de estado a la estrategia: realizado, no realizado, dar seguimiento. Como ya fe mencionada se puede elegir y clasificar el tipo de campaña por perfil o por tipo de evento en el que ya se encuentren inscritos permitiendo que la estrategia de cross selling se aplicable de una manera efectiva.

\section{CONCLUSIONES:}

En un entorno educativo donde la implementación de la educación y de las plataformas digitales es la tendencia y una constante en la industria, es imprescindible lograr adaptar los procesos de las áreas de educación, no solo en el rubor de comunicación con el cliente o medio publicitario, requiere forjarse un entorno facilitador para el cliente cautivo y potencial, ya que de esto dependerá la supervivencia de la marca y preferencia del cliente en nosotros sobre la competencia. 
La manera en la que podamos adaptar los avances tecnológicos, aplicar las estrategias y añadir el toque de personalidad de la marca, ayudaran a encontrar nuevos caminos hacia el posicionamiento y la absorción de más mercado.

La manera en la que la tecnología cambia es verdaderamente impresionante, nadie en esta u otra industria puede quedarse atrás, ya que el tomar esa decisión asegura la extinción de esa marco u organización, existen claros ejemplos de supervivencia en el mercado, así como casos de fracasos rotundos. Pero lo más importante no es el tipo de software, claro, que entre más completo y funcional sea será mejor, lo importante es entender que la filosofía del CRM se basa en el Marketing de Relación, una tendencia que desde los años 90's se expandió a toda las industria, dando un complejo pero claro panorama de que el entender las necesidades, deseos y aspiraciones de los clientes es lo importante, pero, el cómo, es la parte medular que definirá la eficiencia de la marca u organización en esta aspiración.

Como en la mayoría de las estrategias de marketing y más aún, al adaptarse a este tipo de filosofía, hacer entender a nuestros capital humano que son acciones en conjunto, humano -tecnología, pues solo así se podrá cumplir con los objetivos y metas planteados, este siempre ha sido el fin y más importante principio de la innovación tecnológica: apoyar al humano a potencializar sus oportunidades de crecimiento; esto, sin duda es una realidad a la que nos enfrentamos ya que dependerá en su mayoría de la eficiencia y aprovechamiento que muestren los encargados y áreas relacionadas al CRM con respecto a la información que este genere.

\section{REFERENCIAS:}

Blasco T., y Otero, L. (marzo-abril de 2008). Técnicas conversacionales para la recogida de datos en investigación cualitativa: la entrevista. Nure Investigación. (33). Recuperado el 15 de junio del 2019; de https://www.nureinvestigacion.es/OJS/index. php/nure/article/view/408
Córdoba, J. (2009). Del marketing transaccional al marketing relacional. Dialnet. 5 (1). 6-17. Recuperado el 26 de noviembre del 2019 de https://dialnet.unirioja.es/servlet/articulo?codi go $=3993098$

Diaz, M. (14 de Septiembre de 2015). Qué es un CRM y cómo funciona en las empresas. [Blog] Makingexperience. Recuperado el 31 de Octubre de 2019, de https://makingexperience.com/blog/que-esun-crm-y-como-funciona-en-las-empresas/

Espinosa, R. (29 de febrero de 2012). ¿Por qué utilizar estrategias de Cross-selling? Puro Marketing. Recuperado 10 de noviembre del 2019, de http://www.puromarketing.com/13/12324/utili zar-estrategias-crossselling.html

García, I. (2001). CRM: gestión de relación con los clientes. FC Editorial. Madrid, España. Recuperado el 22 de febrero de 2020 de https://books.google.com.mx/books?id=mlAs RGChBc8C\&pg=PA192\&dq=beneficios + de + CRM\&hl=es\&sa=X\&ved=0ahUKEwiRuDwsP PeAhUS11MKHYRUBbcQ6AEIPDAD\#v=one page\&q=beneficios $\% 20 \mathrm{de} \% 20 \mathrm{CRM} \& \mathrm{f}=$ false

Grönroos. (1999). Relationship marketing: challenges for the organization. Journal of Business Research. 46 (3), 327-335. Recuperado el 10 de mayo del 2020 de https://doi.org/10.1016/S01482963(98)00030-7

Grupo 5. (13 de octubre de 2006). CRM Analítico, Operacional y Colaborativo. Grupo5Ulina [Blog]. Recuperado 7 de noviembre de 2019, de

http://grupo05ulima.blogspot.mx/2006/10/crm analtico-operacional-y.html

Harding, F. (2004). Venta Cruzada: Manual de Cross Selling. Ediciciones Gestión 2000. España.

Hernández, S; Fernández, C. \& Baptista, P. (2010). Metodología de la Investigación. Quinta edición. McGraw-Hill / Interamericana Editores. México.

Kotler, P. (2002). Marketing Management: Analysis, Planning, Implementation and Control. $\mathrm{NJ}$ : Prentice Hall 
Martínez, F. \& Luna, P. (2007). Marketing en la sociedad del conocimiento: Claves para la empresa. DELTA Publicaciones. Madrid, España.

Petrella, C. (2008). Gestión de la relación de las universidades con docentes, estudiantes y egresados. Revista Iberoamericana de Educación. Recuperada el 15 de marzo de 2020 de https://doi.org/10.35362/rie4752267

Román, U., Córdova, C. \& Julca, C. (2005). Modelo CRM Para Instituciones Educativas. Revista de investigación de Sistemas e Informática. 2(1). Recuperado el 20 de mayo del 2020 de https://go.gale.com/ps/anonymous?id=GALE $\% 7 C A 172525113 \&$ sid=googleScholar\&v=2.1 \&it=r\&linkaccess=abs\&issn=18163823\& $=\mid \mathrm{F}$ ME\&sw $=W$

Sarmiento, J. (2015). Marketing de relaciones. Aproximación a las relaciones virtuales. Editor: Dykinson .Recuperado el 24 de noviembre del 2019, de https://books.google.com.mx/books?id=IGi7C wAAQBAJ\&pg=PA78\&dq=Grönroos\#v=onep age \&q=Grönroos\&f=false

Valdés, D. P. (26 de Octubre de 2007). ¿Qué son las bases de datos?. Maestros del Web. [Blog] Recuperado el 25 de febrero del 2020 de http://www.maestrosdelweb.com/que-sonlas-bases-de-datos/

$$
\begin{aligned}
& \text { Valle, A., Puerta, A. \& Núñez, R. (2017) Curso } \\
& \text { de Consultoría TIC. Gestión, } \\
& \text { Software ERP y CRM. Editorial IT } \\
& \text { Campus Academy. 2da. Edición. } \\
& \text { Recuperado el } 5 \text { de abril del 2019, de } \\
& \text { https://books.google.com.mx/books?i } \\
& \text { d=SJUSDgAQBAJ\&g=PA1\&dq=v } \\
& \text { alle,+puerta,+nu\%C3\%B1ez+2017\& } \\
& \text { hl=es\&sa=X\&ved=0ahUKEwj0_rSg2 } \\
& \text { vXeAhVJOIMKHdTtAzkQ6AEIKTAA\# } \\
& \text { v=onepage\&q=valle\%2C\%20puerta } \\
& \text { \%2C\%20nu\%C3\%B1ez\%202017\&f= } \\
& \text { false }
\end{aligned}
$$

